PROCEEDINGS OF THE

AMERICAN MATHEMATICAL SOCIETY

Volume 138, Number 1, January 2010, Pages 165-174

S 0002-9939(09)09971-7

Article electronically published on September 3, 2009

\title{
ON APPROXIMATE DIFFERENTIABILITY OF THE MAXIMAL FUNCTION
}

\author{
PIOTR HAJŁASZ AND JAN MALÝ \\ (Communicated by Tatiana Toro) \\ Dedicated to Professor Bogdan Bojarski
}

\begin{abstract}
We prove that if $f \in L^{1}\left(\mathbb{R}^{n}\right)$ is approximately differentiable a.e., then the Hardy-Littlewood maximal function $\mathcal{M} f$ is also approximately differentiable a.e. Moreover, if we only assume that $f \in L^{1}\left(\mathbb{R}^{n}\right)$, then any open set of $\mathbb{R}^{n}$ contains a subset of positive measure such that $\mathcal{M} f$ is approximately differentiable on that set. On the other hand we present an example of $f \in L^{1}(\mathbb{R})$ such that $\mathcal{M} f$ is not approximately differentiable a.e.
\end{abstract}

\section{INTRODUCTION}

Juha Kinnunen [10] proved that the Hardy-Littlewood maximal function

$$
\mathcal{M} f(x)=\sup _{r>0}|B(x, r)|^{-1} \int_{B(x, r)}|f(y)| d y
$$

is a bounded operator in the Sobolev space $W^{1, p}\left(\mathbb{R}^{n}\right), 1<p<\infty$. Recall that $W^{1, p}\left(\mathbb{R}^{n}\right)$ is the space of all functions $f \in L^{p}\left(\mathbb{R}^{n}\right)$ such that weak (distributional) partial derivatives $\partial f / \partial x_{i}$ also belong to $L^{p}\left(\mathbb{R}^{n}\right)$, and similarly for $W_{\text {loc }}^{1, p}\left(\mathbb{R}^{n}\right)$. Since the maximal function is not bounded in $L^{1}$, there is no apparent reason to expect any kind of boundedness of the maximal function in $W^{1,1}\left(\mathbb{R}^{n}\right)$. However, Tanaka 25 proved that in the one dimensional case the noncentered maximal function of $f \in W^{1,1}(\mathbb{R})$ belongs locally to $W^{1,1}(\mathbb{R})$. Since that time it has been an open problem to extend Tanaka's result to the case of the Hardy-Littlewood maximal function and to find analogous results in the higher dimensional case; cf. [8, Question 1]. To the best of our knowledge there are no known higher dimensional results in the case $p=1$, and even in the one dimensional case it is still not known whether the Hardy-Littlewood maximal function (i.e. the centered one) of $f \in W^{1,1}(\mathbb{R})$ belongs locally to $W^{1,1}(\mathbb{R})$; see, however, [2], 3]. The results proved in the paper are clearly motivated by this challenging problem.

Theorem 1. If $f \in L^{1}\left(\mathbb{R}^{n}\right)$ is approximately differentiable a.e., then the maximal function $\mathcal{M} f$ is approximately differentiable a.e.

Received by the editors February 18, 2009.

2000 Mathematics Subject Classification. Primary 42B25; Secondary 46E35, 31B05.

The first author was supported by NSF grant DMS-0500966.

The second author was supported by the research project MSM 0021620839 and by grants GA ČR 201/06/0198, 201/09/0067. 
Since every function $f \in W^{1,1}\left(\mathbb{R}^{n}\right)$ is approximately differentiable a.e., the result implies a.e. approximate differentiability of $\mathcal{M} f$. This, in particular, implies (see Lemma (5) that $\mathcal{M} f$ coincides with a $C^{1}$ function off an open set of arbitrarily small measure. However, a.e. approximate differentiability of $\mathcal{M f}$ is much less than weak differentiability of $\mathcal{M} f$, which is still an open problem. On the other hand, the assumption about $f$ in the theorem is much weaker than $f \in W^{1,1}$. In addition to this result, Theorem 2 provides a formula for the approximate derivative of $\mathcal{M} f$ when $f \in W^{1,1}$.

Let $f \in L^{p}\left(\mathbb{R}^{n}\right), 1 \leq p<\infty$. It is easy to see (cf. 20]) that for a.e. $x \in \mathbb{R}^{n}$, either

or

$$
\mathcal{M} f(x)=f_{B\left(x, r_{x}\right)}|f(y)| d y \quad \text { for some } r_{x}>0
$$

Denote by $E$ and $P$ the sets of points in $\mathbb{R}^{n}$ for which (11) and respectively (2) is satisfied. The following result is due to Luiro [20] when $p>1$ and is new when $p=1$. Our proof is new and simpler even in the case $p>1$.

Theorem 2. Let $f \in W^{1, p}\left(\mathbb{R}^{n}\right), 1 \leq p<\infty$. Then the weak derivative, when $p>1$, and the approximate derivative, when $p=1$, of the maximal function $\mathcal{M} f$ satisfy

$$
\begin{gathered}
\nabla \mathcal{M} f(x)=f_{B\left(x, r_{x}\right)} \nabla|f(y)| d y \quad \text { for a.e. } x \in E, \\
\nabla \mathcal{M} f(x)=\nabla|f(x)| \quad \text { for a.e. } x \in P .
\end{gathered}
$$

Remark 3. If $x \in E$, then $r_{x}>0$ is not necessarily uniquely defined and (3) holds for all such $r_{x}$.

In the next result we deal with differentiability properties of $\mathcal{M} f$ for any $f \in$ $L^{1}\left(\mathbb{R}^{n}\right)$.

Theorem 4. If $f \in L^{1}\left(\mathbb{R}^{n}\right)$, then any open set $\Omega \subset \mathbb{R}^{n}$ contains a subset $E \subset \Omega$ of positive Lebesgue measure such that $\mathcal{M f}$ is approximately differentiable a.e. in E.

Again, Lemma 5 implies that for any open set $\Omega \subset \mathbb{R}^{n}$ there is a function $g \in C^{1}\left(\mathbb{R}^{n}\right)$ such that the set $\{x \in \Omega: f(x)=g(x)\}$ has positive measure.

In view of Theorem 4 it is natural to inquire whether for every $f \in L^{1}\left(\mathbb{R}^{n}\right)$ the maximal function $\mathcal{M} f$ is approximately differentiable a.e. Unfortunately the answer is in the negative, as an example presented at the end of the paper shows.

While the proofs of Theorems 1 and 2 are completely elementary, the proof of Theorem 4 requires some advanced potential theory.

Let us also mention that the result of Kinnunen 10, has been applied and gen-

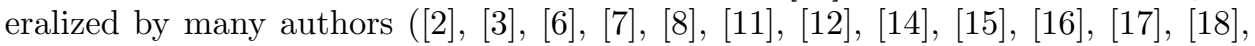
20], 21], 25]).

The notation used in the paper is pretty standard. The volume of the unit ball in $\mathbb{R}^{n}$ is denoted by $\omega_{n}$, and we use a barred integral to denote the integral average

$$
f_{B(x, r)} f(y) d y=\frac{1}{|B(x, r)|} \int_{B(x, r)} f(y) d y .
$$


By $C$ we will denote a generic positive constant whose actual value may change even in a single string of estimates.

\section{Approximate Differentiability}

Let $f$ be a real-valued function defined on a set $E \subset \mathbb{R}^{n}$. We say that $f$ is approximately differentiable at $x_{0} \in E$ if there is a vector $L=\left(L_{1}, \ldots, L_{n}\right)$ such that for any $\varepsilon>0$ the set

$$
A_{\varepsilon}=\left\{x: \frac{\left|f(x)-f\left(x_{0}\right)-L\left(x-x_{0}\right)\right|}{\left|x-x_{0}\right|}<\varepsilon\right\}
$$

has $x_{0}$ as a density point. If this is the case, then $x_{0}$ is a density point of $E$ and $L$ is uniquely determined. The vector $L$ is called the approximate differential of $f$ at $x_{0}$ and is denoted by $\nabla f\left(x_{0}\right)$.

In what follows we will need the following theorem of Whitney [26, which provides several characterizations of a.e. approximate differentiability of a function. We state it as a lemma.

Lemma 5. Let $f: E \rightarrow \mathbb{R}$ be measurable, $E \subset \mathbb{R}^{n}$. Then the following conditions are equivalent.

(a) $f$ is approximately differentiable a.e.

(b) For any $\varepsilon>0$ there is a closed set $F \subset E$ and a locally Lipschitz function $f: \mathbb{R}^{n} \rightarrow \mathbb{R}$ such that $\left.f\right|_{F}=\left.g\right|_{F}$ and $|E \backslash F|<\varepsilon$.

(c) For any $\varepsilon>0$ there is a closed set $F \subset E$ and a function $g \in C^{1}\left(\mathbb{R}^{n}\right)$ such that $\left.f\right|_{F}=\left.g\right|_{F}$ and $|E \backslash F|<\varepsilon$.

Remark 6. To illustrate the relevance of the maximal function in this part of the real analysis, let us mention a useful Lipschitz type estimate valid for Sobolev functions:

$$
|f(x)-f(y)| \leq C|x-y|(\mathcal{M}|\nabla f|(x)+\mathcal{M}|\nabla f|(y)) \quad \text { a.e.; }
$$

see [1, 4], 5]. As an almost immediate consequence of (4) one obtains a well known result that each $f \in W_{\text {loc }}^{1,1}\left(\mathbb{R}^{n}\right)$ is approximately differentiable a.e.; cf. [22].

Investigating the positive and negative parts of a function separately, one can easily prove

Lemma 7. A measurable function $f: E \rightarrow \mathbb{R}$ is a.e. approximately differentiable if and only if $|f|$ is a.e. approximately differentiable.

\section{Proof of Theorem 1}

We consider a restricted version of the maximal function

$$
\mathcal{M}^{\varepsilon} f(x)=\sup _{r \geq \varepsilon} f_{B(x, r)}|f(y)| d y .
$$

Lemma 8. If $f \in L^{1}\left(\mathbb{R}^{n}\right)$, then

$$
\left|\mathcal{M}^{\varepsilon} f(x)-\mathcal{M}^{\varepsilon} f(y)\right| \leq \frac{n}{\varepsilon}|x-y|\left(\mathcal{M}^{\varepsilon} f(x)+\mathcal{M}^{\varepsilon} f(y)\right) \leq \frac{2 n}{\omega_{n} \varepsilon^{n+1}}\|f\|_{1}|x-y| .
$$


Proof. The second inequality of the lemma is obvious because

$$
\mathcal{M}^{\varepsilon} f(x) \leq \frac{1}{\omega_{n} \varepsilon^{n}} \int_{\mathbb{R}^{n}}|f(y)| d y=\frac{1}{\omega_{n} \varepsilon^{n}}\|f\|_{1} .
$$

Thus we are left with the proof of the first inequality. For $a, r>0$ the function $\varphi(r)=r /(r+a)$ is increasing, and hence, applying Bernoulli's inequality, we have for $r \geq \varepsilon$,

$$
\left(\frac{r}{r+|x-y|}\right)^{n} \geq\left(\frac{\varepsilon}{\varepsilon+|x-y|}\right)^{n} \geq 1-n \frac{|x-y| / \varepsilon}{1+|x-y| / \varepsilon} \geq 1-\frac{n}{\varepsilon}|x-y| .
$$

Fix $x, y \in \mathbb{R}^{n}$. Then for any $r \geq \varepsilon$ we have $B(y, r) \subset B(x, r+|x-y|)$ and hence

$$
\mathcal{M}^{\varepsilon} f(x) \geq\left(\frac{r}{r+|x-y|}\right)^{n} f_{B(y, r)}|f| \geq\left(1-\frac{n}{\varepsilon}|x-y|\right) f_{B(y, r)}|f| .
$$

Passing to the supremum over $r \geq \varepsilon$ we obtain

$$
\mathcal{M}^{\varepsilon} f(x) \geq\left(1-\frac{n}{\varepsilon}|x-y|\right) \mathcal{M}^{\varepsilon} f(y) .
$$

Since the inequality is also true if we replace $x$ by $y$ and $y$ by $x$, one easily concludes the first inequality from the lemma.

Lemma 9. If $f \in L^{1}\left(\mathbb{R}^{n}\right)$, then

$$
\{x: \mathcal{M} f(x)>|f(x)|\}=Z \cup \bigcup_{k=1}^{\infty} E_{k},
$$

where $|Z|=0$ and $\left.\mathcal{M} f\right|_{E_{k}}$ is Lipschitz continuous for $k=1,2, \ldots$ In particular $\mathcal{M} f$ is a.e. approximately diferentiable in the set $\{x: \mathcal{M} f(x)>|f(x)|\}$.

Proof. Let $Z$ be the set of points that are not Lebesgue points of $|f|$. Clearly $|Z|=0$. Assume that $x \in \mathbb{R}^{n} \backslash Z$ and $\mathcal{M} f(x)>|f(x)|$. Let $r_{i}>0$ be a sequence such that

$$
f_{B\left(x_{i}, r_{i}\right)}|f| \rightarrow \mathcal{M} f(x) .
$$

The sequence $r_{i}$ is bounded (because $\mathcal{M} f(x)>0$ and $f \in L^{1}$ ), and hence we can select a subsequence (still denoted by $r_{i}$ ) such that $r_{i} \rightarrow r$. Clearly $r>0$ as otherwise we would have $\mathcal{M} f(x)=|f(x)|$. Thus

$$
\mathcal{M} f(x)=f_{B(x, r)}|f| \text { for some } r>0 .
$$

This easily implies that

$$
\{x: \mathcal{M} f(x)>|f(x)|\} \subset Z \cup \bigcup_{k=1}^{\infty}\left\{x: \mathcal{M} f(x)=\mathcal{M}^{1 / k} f(x)\right\} .
$$

Since the function $\mathcal{M}^{1 / k} f$ is Lipschitz continuous by Lemma 8 , the first part of the result follows. The second part is a direct consequence of Lemma 5 .

Now we can complete the proof of Theorem 1, Let $f \in L^{1}\left(\mathbb{R}^{n}\right)$ be approximately differentiable a.e. Then also $|f|$ is approximately differentiable a.e. (Lemma 7). According to Lemma 9 ,

$$
\mathbb{R}^{n}=\{x: \mathcal{M} f(x)=|f(x)|\} \cup Z \cup \bigcup_{k=1}^{\infty} E_{k},
$$


where $|Z|=0$ and $\left.\mathcal{M} f\right|_{E_{k}}$ is Lipschitz continuous. Since $\left.\mathcal{M} f\right|_{E_{k}}$ is approximately differentiable a.e. and $\mathcal{M} f=|f|$ is approximately differentiable a.e. in the set $\{x: \mathcal{M} f(x)=|f(x)|\}$, the theorem follows.

\section{Proof of Theorem 2}

Since $\mathcal{M} f(x)=|f(x)|$ in $P$, clearly $\nabla \mathcal{M} f(x)=\nabla|f(x)|$ a.e. in $P$. Thus let $x \in E$ and $r_{x}>0$ be such that equality (11) holds. Assume also that $\mathcal{M} f$ is approximately differentiable at $x$. Note that the function

$$
\varphi(y)=\mathcal{M} f(y)-f_{B\left(y, r_{x}\right)}|f(z)| d z=\mathcal{M} f(y)-f_{B\left(0, r_{x}\right)}|f(y+z)| d z
$$

is approximately differentiable at $x$ and

$$
\begin{aligned}
\nabla \varphi(x) & =\nabla \mathcal{M} f(x)-f_{B\left(0, r_{x}\right)} \nabla|f|(x+z) d z \\
& =\nabla \mathcal{M} f(x)-f_{B\left(x, r_{x}\right)} \nabla|f(z)| d z .
\end{aligned}
$$

Indeed, $\mathcal{M} f$ is approximately differentiable at $x$, and since $f \in W^{1, p}$ we can differentiate in the second term under the sign of the integral. Note also that $\varphi \geq 0$ and $\varphi(x)=0$, so $\varphi$ attains a minimum at $x$, and hence its approximate derivative at $x$ must be equal to 0 , which is the claim we wanted to prove.

\section{Proof of Theorem 4}

This proof requires some results from potential theory. We say that a locally integrable function $u: \Omega \rightarrow[0, \infty]$ defined on an open set $\Omega \subset \mathbb{R}^{n}$ is superharmonic if it is lower semicontinuous and

$$
u(x) \geq f_{B(x, r)} u(y) d y
$$

whenever $B(x, r) \Subset \Omega$.

The following regularity result has been established in the setting of weak solutions of the $p$-Laplace equation in 19. For the convenience of the reader, we include a short proof based only on a knowledge of classical potential theory.

Lemma 10. If a locally integrable function $u: \Omega \rightarrow[0, \infty], \Omega \subset \mathbb{R}^{n}$, is superharmonic, then $u \in W_{\text {loc }}^{1, p}(\Omega)$ for all $1 \leq p<n /(n-1)$. In particular $u$ is a.e. approximately differentiable.

Proof. Let $u: \Omega \rightarrow[0, \infty]$ be superharmonic and let $U \Subset \Omega$. According to the Riesz decomposition theorem [23, [9], $u$ restricted to $U$ can be represented as

$$
u(x)=h(x)-\int_{\mathbb{R}^{n}} \Phi(x-y) d \mu(y), \quad \text { for } x \in U,
$$

where $h$ is harmonic, $\Phi$ is the fundamental solution to the Laplace equation and $\mu$ is a finite positive measure supported in $U$. It is easy to see that we can compute the weak first-order partial derivatives of $u$ in $U$ by differentiating the right-hand side of (6) under the sign of the integral

$$
\frac{\partial u}{\partial x_{i}}(x)=\frac{\partial h}{\partial x_{i}}(x)-\frac{1}{n \omega_{n}} \int_{\mathbb{R}^{n}} \frac{\left(x_{i}-y_{i}\right)}{|x-y|^{n}} d \mu(y) .
$$


By Young's convolution inequality (cf. [24, II.1.1, p. 27]), the convolution is as integrable as the kernel. Since the measure $\mu$ has a bounded support and the function $x \mapsto \frac{x}{|x|^{n}}$ is clearly in $L_{\mathrm{loc}}^{p}\left(\mathbb{R}^{n}\right)$, we deduce that $\nabla u \in L^{p}(U)$.

For an open set $\Omega \subset \mathbb{R}^{n}$ and $f \in L_{\text {loc }}^{1}(\Omega)$ we define a local maximal function

$$
\mathcal{M}_{\Omega} f(x)=\sup f_{B(x, r)}|f(y)| d y,
$$

where the supremum is over all balls $B(x, r) \Subset \Omega$.

The following characterization of superharmonic functions will be very useful; see 13 .

Lemma 11. A locally integrable function $u: \Omega \rightarrow[0, \infty], \Omega \subset \mathbb{R}^{n}$, is superharmonic if and only if

$$
\mathcal{M}_{\Omega} u(x)=u(x) \quad \text { for all } x \in \Omega .
$$

Proof. If $u$ is superharmonic, then taking the supremum over all balls in (5) gives $u(x) \geq \mathcal{M}_{\Omega} u(x)$ for all $x \in \Omega$. On the other hand, lower semicontinuity of $u$ yields

$$
\mathcal{M}_{\Omega} u(x) \geq \limsup _{r \rightarrow 0} f_{B(x, r)} u(y) d y \geq \liminf _{y \rightarrow x} u(y) \geq u(x)
$$

for all $x \in \Omega$. Hence $u(x)=\mathcal{M}_{\Omega} u(x)$ for all $x \in \Omega$.

Now suppose that $\mathcal{M}_{\Omega} u(x)=u(x)$ for all $x \in \Omega$. Since the maximal function is lower semicontinuous we conclude lower semicontinuity of $u$. The superharmonicity of $u$ follows from the inequality

$$
f_{B(x, r)} u(y) d y \leq \mathcal{M}_{\Omega} u(x)=u(x),
$$

which is satisfied on every ball $B(x, r) \Subset \Omega$.

Corollary 12. If $f \in L^{1}\left(\mathbb{R}^{n}\right)$ and $|f(x)|=\mathcal{M} f(x)$ a.e. in an open set $\Omega \subset \mathbb{R}^{n}$, then we can redefine $f$ on a set of measure zero in such a way that $|f|$ becomes superharmonic in $\Omega$.

Proof. It follows from the Lebesgue differentiation theorem that $|f(x)| \leq \mathcal{M}_{\Omega}|f|(x)$ a.e. in $\Omega$. Hence

$$
\mathcal{M}_{\Omega}|f|(x) \leq \mathcal{M}|f|(x)=\mathcal{M} f(x)=|f(x)| \leq \mathcal{M}_{\Omega}|f|(x)
$$

a.e. in $\Omega$ and thus $|f(x)|=\mathcal{M}_{\Omega}|f|(x)$ a.e. in $\Omega$. Now it is clear that we can modify $f$ on a set of measure zero in such a way that

$$
|f(x)|=\mathcal{M}_{\Omega}|f|(x) \quad \text { everywhere in } \Omega,
$$

which makes the function $|f|$ superharmonic.

Now we can complete the proof of the theorem. Let $f \in L^{1}\left(\mathbb{R}^{n}\right)$ and let $\Omega \subset \mathbb{R}^{n}$ be open. According to Lemma $9, \mathcal{M} f$ is a.e. approximately differentiable in the set

$$
\{x \in \Omega:|f(x)|<\mathcal{M} f(x)\} .
$$

If this set has positive measure, the theorem follows. If it has measure zero, then

$$
|f(x)|=\mathcal{M} f(x) \quad \text { a.e. in } \Omega,
$$

and hence $|f(x)|$ coincides a.e. with a superharmonic function in $\Omega$; see Corollary 12. Now Lemma 10 gives a.e. approximate differentiability of $|f|$ in $\Omega$ and hence that of $f$; see Lemma 7 , The proof is complete. 


\section{EXAMPLE}

In this section we will construct a bounded integrable function $f \in L^{1}(\mathbb{R})$ such that the set of points where the maximal function $\mathcal{M} f$ is not approximately differentiable is of positive measure. In our construction $\mathcal{M} f$ will coincide with $f$ on a contact set $P$ of positive length, and $f$ will not be approximately differentiable on $P$. This will imply the lack of approximate differentiability of $\mathcal{M} f$ at the Lebesgue points of $P$.

In the first step we will construct a bounded periodic function $f$ with period 1 such that $\mathcal{M} f$ is not approximately differentiable a.e., and then it will be clear that also for $\tilde{f}=f \chi_{[0,1]} \in L^{1}(\mathbb{R})$ the maximal function is not approximately differentiable a.e.

6.1. Construction. We denote

$$
r_{k}=3^{-k(k+1)}, \quad \alpha_{k}=\exp \left(-9^{-k-2}\right) .
$$

For $k=1,2, \ldots$, on the interval $\left[0, r_{k-1}\right)$ we define

$$
g_{k}(x)=\left\{\begin{array}{lll}
1, & x \in\left[(i-1) r_{k}, i r_{k}\right), & i \in\left\{2,4, \ldots, 9^{k}-1\right\}, \\
\alpha_{k}, & x \in\left[(i-1) r_{k}, i r_{k}\right), & i \in\left\{3,5, \ldots, 9^{k}-2\right\}, \\
0, & x \in\left[(i-1) r_{k}, i r_{k}\right), & i \in\left\{1,9^{k}\right\} .
\end{array}\right.
$$

We extend $g_{k}$ to $\mathbb{R}$ periodically with the period $r_{k-1}$. Finally we set

$$
f_{0}=1, \quad f_{n}=\prod_{k=1}^{n} g_{k}, \quad f=\lim _{n \rightarrow \infty} f_{n} .
$$

Observe that the function $g_{k}$ is constant on the intervals $\left[(i-1) r_{k}, i r_{k}\right), i \in \mathbb{Z}$, and hence $f_{n}$ is constant on the intervals $\left[(i-1) r_{n}, i r_{n}\right), i \in \mathbb{Z}$.

6.2. Maximal function. We will now estimate the maximal function of $f$. We denote

$$
P=\bigcap_{k}\left\{f_{k}>0\right\} \text {. }
$$

Let $x \in \mathbb{R}$ and $\rho>0$. We consider the smallest $n \in \mathbb{N}$ such that $M_{n}(x, \rho)=$ $(x-\rho, x+\rho) \cap r_{n} \mathbb{Z} \neq \emptyset$. In this situation $(x-\rho, x+\rho)$ is contained in one of the intervals $\left[(i-1) r_{n-1}, i r_{n-1}\right)$, and hence $f_{n-1}$ equals a constant $\beta$ on $(x-\rho, x+\rho)$. Let us write $M=M_{n}(x, \rho)$. Now we will distinguish two cases.

Case 1. Let $\rho \leq r_{n+1}$. Then there is only one point $z \in M$. By reason of symmetry, we may assume that $x \geq z$. Then $x \in\left[z, z+r_{n+1}\right)$. Since $g_{n+1}=0$ on $\left[z, z+r_{n+1}\right), f_{n+1}=0$ on that interval and hence $x \notin P$.

Case 2. Let $\rho>r_{n+1}$. We split $(x-\rho, x+\rho)$ into intervals $(x-\rho, x+\rho) \cap$ $\left[(i-1) r_{n}, i r_{n}\right)$. For each interval $I$ of the partition, with an endpoint $z \in M$, either $\left[z, z+r_{n+1}\right) \subset I,\left[z-r_{n+1}, z\right) \subset I$ or $I \subset\left(z-r_{n+1}, z+r_{n+1}\right)$. Since $f_{n+1}=0$ on $\left(z-r_{n+1}, z+r_{n+1}\right)$ we have $\left(z-r_{n+1}, z+r_{n+1}\right) \cap P=\emptyset$. In each case

$$
|I \cap P| \leq\left(1-\frac{r_{n+1}}{r_{n}}\right)|I| .
$$

Summing over $I$ we obtain

$$
|(x-\rho, x+\rho) \cap P| \leq 2 \rho\left(1-\frac{r_{n+1}}{r_{n}}\right) .
$$


It follows that

$$
f_{x-\rho}^{x+\rho} f \leq \frac{\beta}{2 \rho}|(x-\rho, x+\rho) \cap P| \leq \beta\left(1-\frac{r_{n+1}}{r_{n}}\right) .
$$

On the other hand, if $x \in P$, then

$$
f(x) \geq \beta \alpha_{n} \alpha_{n+1} \ldots
$$

Since

$$
1-\frac{r_{n+1}}{r_{n}}=1-9^{-n-1} \leq e^{-9^{-n-1}}<e^{-9^{-n-2}-9^{-n-3}-9^{-n-4}-\ldots}=\alpha_{n} \alpha_{n+1} \ldots,
$$

we obtain

$$
\mathcal{M} f(x) \leq f(x) \quad \text { on } P
$$

and hence $\mathcal{M} f(x)=f(x)$ a.e. in $P$.

6.3. The contact set. On the set $P$ we have

$$
f(x) \geq \beta_{\infty}:=\alpha_{1} \alpha_{2} \alpha_{3} \cdots=\exp \left(-9^{-3}-9^{-4}-9^{-5}-\ldots\right)>0 .
$$

We will estimate the size of the set $P \cap[0,1]$. We see that

$$
\begin{gathered}
\left|\left\{f_{1}>0\right\} \cap[0,1]\right|=1-2 r_{1}, \\
\left|\left\{f_{2}>0\right\} \cap[0,1]\right|=\left|\left\{f_{1}>0\right\} \cap[0,1]\right|\left(1-2 \frac{r_{2}}{r_{1}}\right)=\left(1-2 r_{1}\right)\left(1-2 \frac{r_{2}}{r_{1}}\right), \\
\ldots,
\end{gathered}
$$

so that

$$
|P \cap[0,1]|=\left(1-2 r_{1}\right)\left(1-2 \frac{r_{2}}{r_{1}}\right)\left(1-2 \frac{r_{3}}{r_{2}}\right) \cdots>0
$$

as

$$
\sum_{k} \frac{r_{k+1}}{r_{k}}=\sum_{k} 9^{-k-1}<+\infty .
$$

6.4. Differentiability. Let us consider $x \in P, k \in \mathbb{N}$ and an interval $\left[z, z+r_{k}\right)$ such that $z \in r_{k} \mathbb{Z}$ and

$$
x \in\left[z, z+r_{k}\right) \subset\left\{f_{k}>0\right\} .
$$

This interval is contained in an interval $\left[(i-1) r_{k-1}, i r_{k-1}\right)$, where the function $f_{k-1}$ has constant value $\beta \in\left(\beta_{\infty}, 1\right]$. Since $z \geq(i-1) r_{k-1}+r_{k}$ (otherwise $\left.f_{k}(z)=0\right)$ we have that

$$
\left[z-r_{k}, z+r_{k}\right) \subset\left[(i-1) r_{k-1}, i r_{k-1}\right)
$$

and hence $f_{k-1}=\beta$ on $\left[z-r_{k}, z+r_{k}\right)$. There are three possibilities:

In each case

$$
\begin{gathered}
f_{k}= \begin{cases}0 & \text { on }\left[z-r_{k}, z\right)=I, \\
\beta & \text { on }\left[z, z+r_{k}\right)=J,\end{cases} \\
f_{k}= \begin{cases}\beta & \text { on }\left[z-r_{k}, z\right)=J, \\
\alpha_{k} \beta & \text { on }\left[z, z+r_{k}\right)=I,\end{cases} \\
f_{k}= \begin{cases}\alpha_{k} \beta & \text { on }\left[z-r_{k}, z\right)=I, \\
\beta & \text { on }\left[z, z+r_{k}\right)=J .\end{cases}
\end{gathered}
$$

$$
f \leq \beta \alpha_{k}=\beta \exp \left(-9^{-k-2}\right) \quad \text { on } I
$$


whereas

$$
f \geq \beta \alpha_{k+1} \alpha_{k+2} \alpha_{k+3} \ldots=\beta \exp \left(-\frac{9^{-k-2}}{8}\right) \quad \text { on } J \cap P .
$$

Hence

$$
|f-f(x)| \geq \frac{1}{2} \beta\left(\exp \left(-\frac{9^{-k-2}}{8}\right)-\exp \left(-9^{-k-2}\right)\right)
$$

on at least one of the sets $I$ or $J \cap P$. Since the infinite product at (7) converges, for sufficiently large $k$ we have

$$
|J \cap P|=|J|\left(1-2 \frac{r_{k+1}}{r_{k}}\right)\left(1-2 \frac{r_{k+2}}{r_{k+1}}\right) \cdots>\frac{1}{2}|J|=\frac{r_{k}}{2},
$$

and hence inequality (8) is satisfied on a set $E_{k} \subset\left[z-r_{k}, z+r_{k}\right)$ of length $\left|E_{k}\right|$ $>r_{k} / 2$. To estimate the right hand side of (8), observe that $e^{-x}-e^{-y} \geq$ $e^{-y}\left(e^{y-x}-1\right) \geq(1-y)(y-x), 0<x<y$, and thus

$$
\exp \left(-\frac{9^{-k-2}}{8}\right)-\exp \left(-9^{-k-2}\right) \geq \frac{7}{8}\left(1-9^{-k-2}\right) 9^{-k-2}>4 \cdot 9^{-k-3} .
$$

Accordingly

$$
\frac{|f(y)-f(x)|}{|y-x|} \geq \frac{4 \beta 9^{-k-3}}{4 r_{k}}=\beta 3^{k^{2}-k-6} \quad \text { for } y \in E_{k} .
$$

Set $E_{n}^{*}=\bigcup_{k=n}^{\infty} E_{k}$. Since

$$
\limsup _{h \rightarrow 0+} \frac{\left|E_{n}^{*} \cap(x-h, x+h)\right|}{2 h} \geq \limsup _{k \rightarrow \infty} \frac{\left|E_{k}\right|}{4 r_{k}} \geq \frac{1}{8},
$$

the approximate limit-superior of $\frac{|f(y)-f(x)|}{|y-x|}$ as $y \rightarrow x$ is at least $\beta 3^{n^{2}-n-6}$ for each $n$, and thus it is $\infty$. Hence $f$ cannot be approximately differentiable at $x$. If $x$ is a density point of $P$, then also $\mathcal{M} f$ cannot be approximately differentiable at $x$.

6.5. An integrable function. Finally let $\tilde{f}=f \chi_{(0,1)}$. Then $\mathcal{M} \tilde{f}(x) \leq \mathcal{M} f(x) \leq$ $f(x)=\tilde{f}(x)$ in $P \cap(0,1)$ and hence $\mathcal{M} \tilde{f}(x)=\tilde{f}(x)=f(x)$ a.e. in $P \cap(0,1)$. Since $f$ is not approximately differentiable on $P \cap(0,1), \mathcal{M} \tilde{f}$ cannot be approximately differentiable a.e.

\section{REFERENCES}

[1] Acerbi, E., Fusco, N.: An approximation lemma for $W^{1, p}$ functions. In: Material instabilities in continuum mechanics (Edinburgh, 1985-1986), pp. 1-5, Oxford Sci. Publ., Oxford Univ. Press, New York, 1988. MR970512 (89m:46060)

[2] Aldaz, J. M., Pérez Lázaro, J.: Boundedness and unboundedness results for some maximal operators on functions of bounded variation. J. Math. Anal. Appl. 337 (2008), 130-143. MR2356061 (2008k:42050)

[3] Aldaz, J. M., Pérez Lázaro, J.: Functions of bounded variation, the derivative of the one dimensional maximal function, and applications to inequalities. Trans. Amer. Math. Soc. 359 (2007), 2443-2461. MR2276629 (2008f:42010)

[4] Bojarski, B.: Remarks on some geometric properties of Sobolev mappings. In: Functional analysis $\&$ related topics (Sapporo, 1990), pp. 65-76, World Sci. Publ., River Edge, NJ, 1991. MR.1148607 (92k:46046)

[5] Bojarski, B., Hajłasz, P.: Pointwise inequalities for Sobolev functions and some applications. Studia Math. 106 (1993), 77-92. MR:1226425 (94h:46045)

[6] Buckley, S. M.: Is the maximal function of a Lipschitz function continuous? Ann. Acad. Sci. Fenn. Math. 24 (1999), 519-528. MR.1724375(2001e:42025) 
[7] Carneiro, E., Moreira, D.: On the regularity of maximal operators. Proc. Amer. Math. Soc. 136 (2008), 4395-4404. MR2431055

[8] Hajłasz, P., Onninen, J.: On boundedness of maximal functions in Sobolev spaces. Ann. Acad. Sci. Fenn. Math. 29 (2004), 167-176. MR2041705 (2005a:42010)

[9] Hayman, W. K., Kennedy, P. B.: Subharmonic functions. Vol. I. London Mathematical Society Monographs, No. 9. Academic Press, London-New York, 1976. MR0460672 (57:665)

[10] Kinnunen, J.: The Hardy-Littlewood maximal function of a Sobolev function. Israel J. Math. 100 (1997), 117-124. MR1469106 (99a:30029)

[11] Kinnunen, J., Latvala, V.: Lebesgue points for Sobolev functions on metric spaces. Rev. Mat. Iberoamericana 18 (2002), 685-700. MR 1954868 (2004c:46054)

[12] Kinnunen, J., Lindqvist, P.: The derivative of the maximal function. J. Reine Angew. Math. 503 (1998), 161-167. MR.1650343 (99j:42027)

[13] Kinnunen, J., Martio, O.: Maximal operator and superharmonicity. In: Function spaces, differential operators and nonlinear analysis (Pudasjärvi, 1999), pp. 157-169, Acad. Sci. Czech Repub., Prague, 2000. MR.1755307 (2001f:31005)

[14] Kinnunen, J., Saksman, E.: Regularity of the fractional maximal function. Bull. London Math. Soc. 35 (2003), 529-535. MR 1979008(2004e:42035)

[15] Kinnunen, J., Tuominen, H.: Pointwise behaviour of $M^{1,1}$ Sobolev functions. Math. Z. 257 (2007), 613-630. MR2328816(2008e:46042)

[16] Korry, S.: A class of bounded operators on Sobolev spaces. Arch. Math. (Basel) 82 (2004), 40-50. MR2034469 (2004k:42033)

[17] Korry, S.: Extensions of Meyers-Ziemer results. Israel J. Math. 133 (2003), 357-367. MR1968435 (2004c:46055)

[18] Korry, S.: Boundedness of Hardy-Littlewood maximal operator in the framework of LizorkinTriebel spaces. Rev. Mat. Complut. 15 (2002), 401-416. MR.1951818 (2004a:42020)

[19] Lindqvist, P.: On the definition and properties of $p$-superharmonic functions. J. Reine Angew. Math. 365 (1986), 67-79. MR826152(87e:31011)

[20] Luiro, H.: Continuity of the maximal operator in Sobolev spaces. Proc. Amer. Math. Soc. 135 (2007), 243-251. MR 2280193 (2007i:42021)

[21] MacManus, P.: Poincaré inequalities and Sobolev spaces. In: Proceedings of the 6th International Conference on Harmonic Analysis and Partial Differential Equations (El Escorial, 2000). Publ. Mat. 2002, Vol. Extra, pp. 181-197. MR.1964820 (2004j:46049)

[22] Malý, J., Ziemer, W. P.: Fine regularity of solutions of elliptic partial differential equations. Mathematical Surveys and Monographs, 51. American Mathematical Society, Providence, RI, 1997. MR.1461542 (98h:35080)

[23] Ransford, T.: Potential theory in the complex plane. London Mathematical Society Student Texts, 28. Cambridge University Press, Cambridge, 1995. MR.1334766 (96e:31001)

[24] Stein, E. M.: Singular integrals and differentiability properties of functions. Princeton Mathematical Series, No. 30. Princeton University Press, Princeton, N.J., 1970. MR0290095 $(44: 7280)$

[25] Tanaka, H.: A remark on the derivative of the one-dimensional Hardy-Littlewood maximal function. Bull. Austral. Math. Soc. 65 (2002), 253-258. MR.1898539(2002m:42017)

[26] Whitney, H.: On totally differentiable and smooth functions. Pacific J. Math. 1 (1951), 143-159. MR0043878(13:333d)

Department of Mathematics, University of Pittsburgh, 301 Thackeray Hall, PittsBurgh, Pennsylvania 15260

E-mail address: hajlasz@pitt.edu

Department KMA of the Faculty of Mathematics and Physics, Charles University, Sokolovská 83, CZ-18675 Praha 8, Czech Republic - and - Department of Mathematics of the Faculty of Science, J. E. Purkyně University, České mládeže 8, 40096 Ústí nad Labem, Czech Republic

E-mail address: maly@karlin.mff.cuni.cz 